\title{
Article
}

\section{Secondary fast magnetoacoustic waves trapped in randomly structured plasmas}

\author{
Yuan, D, Li, B and Walsh, RW \\ Available at http://clok.uclan.ac.uk/15366/ \\ Yuan, D ORCID: 0000-0002-9514-6402, Li, B and Walsh, RW ORCID: 0000- \\ 0002-1025-9863 (2016) Secondary fast magnetoacoustic waves trapped in \\ randomly structured plasmas. The Astrophysical Journal, 828 (1). ISSN $0004-$ \\ $637 x$
}

It is advisable to refer to the publisher's version if you intend to cite from the work. http://dx.doi.org/10.3847/0004-637X/828/1/17

For more information about UCLan's research in this area go to http://www.uclan.ac.uk/researchgroups/ and search for < name of research Group>.

For information about Research generally at UCLan please go to http://www.uclan.ac.uk/research/

All outputs in CLoK are protected by Intellectual Property Rights law, including Copyright law. Copyright, IPR and Moral Rights for the works on this site are retained by the individual authors and/or other copyright owners. Terms and conditions for use of this material are defined in the policies page.

\section{CLoK}

Central Lancashire online Knowledge www.clok.uclan.ac.uk

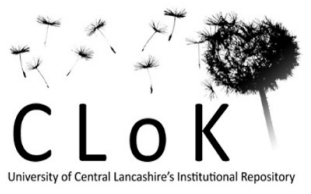




\title{
Secondary fast magnetoacoustic waves trapped in randomly structured plasmas
}

\author{
Ding Yuan ${ }^{1,2}$ \\ DYuan2@uclan.ac.uk \\ Bo $\mathrm{Li}^{3}$ \\ and \\ Robert W. Walsh ${ }^{1}$
}

\begin{abstract}
Fast magnetoacoustic wave is an important tool for inferring solar atmospheric parameters. We numerically simulate the propagation of fast wave pulses in randomly structured plasmas mimicking the highly inhomogeneous solar corona. A network of secondary waves is formed by a series of partial reflections and transmissions. These secondary waves exhibit quasi-periodicities in both time and space. Since the temporal and spatial periods are related simply through the fast wave speed, we quantify the properties of secondary waves by examining the dependence of the average temporal period $(\bar{p})$ on the initial pulse width $\left(w_{0}\right)$ as well as the density contrast $\left(\delta_{\rho}\right)$ and correlation length $\left(L_{c}\right)$ that characterize the randomness of the equilibrium density profiles. For small-amplitude pulses, $\delta_{\rho}$ does not alter $\bar{p}$ significantly. Large-amplitude pulses, on the other hand, enhance the density contrast when $\delta_{\rho}$ is small but have a smoothing effect when $\delta_{\rho}$ is sufficiently large. We found that $\bar{p}$ scales linearly with $L_{c}$ and that the scaling factor is larger for a narrower pulse. However, in terms of the absolute values of $\bar{p}$, broader pulses generate secondary waves with longer periods, and this effect is stronger in random plasmas with shorter correlation lengths. Secondary waves carry the signatures of both the leading wave pulse and background plasma, our study may find applications in MHD seismology by exploiting the secondary waves detected in the dimming regions after CMEs or EUV waves.
\end{abstract}

Subject headings: Sun: atmosphere - Sun: corona - Sun: oscillations - magnetohydrodynamics (MHD) - methods: numerical — waves

\footnotetext{
${ }^{1}$ Jeremiah Horrocks Institute, University of Central Lancashire, Preston, PR1 2HE, United Kingdom

${ }^{2}$ Key Laboratory of Solar Activity, National Astronomical Observatories, Chinese Academy of Sciences, Beijing, 100012

${ }^{3}$ Shandong Provincial Key Laboratory of Optical Astronomy and Solar-Terrestrial Environment, Institute of Space Sciences, Shandong University, Weihai, 264209 Shandong, China
}

\section{Introduction}

Fast magnetohydrodynamic (MHD) waves can propagate across various magnetic structures, and could therefore be easily trapped in structures with low Alfvén speeds (see e.g., Goedbloed \& Poedts 2004). Plasma structuring modifies how MHD waves propagate and leads to interesting effects such as wave-guiding, dispersion, mode coupling, resonant absorption, and phase mixing (Edwin \& Roberts 1983; Van Doorsselaere et al. 2008; Sakurai et al. 1991; Heyvaerts \& Priest 1983). 
Theoretical studies on MHD waves in structured plasmas, combined with the abundant measurements of low-frequency waves and oscillations in the solar atmosphere, can be employed to infer the solar atmospheric parameters that are difficult to measure directly (see the reviews by Nakariakov \& Verwichte 2005; De Moortel \& Nakariakov 2012, and references therein). This technique, commonly referred to as solar MHD seismology, has been successful in yielding the magnetic field strength (Nakariakov \& Ofman 2001), plasma beta (Zhang et al. 2015), transverse structuring (Aschwanden et al. 2003), and longitudinal Alfvén transit time (Arregui et al. 2007) in various coronal loops. In addition, it has also been adopted to infer the effective polytropic index of coronal plasmas (Van Doorsselaere et al. 2011), magnetic topology of sunspots (Yuan et al. 2014b,a), and the magnetic structure of large-scale coronal streamers (Chen et al. 2010, 2011).

While not a common practice in the literature, modelling the inhomogeneous solar atmosphere as randomly structured plasmas is more appropriate in a number of situations. For instance, this approach has been adopted to model the plethora of thin fibrils in sunspots (Keppens et al. 1994), the filamentary coronal loops (Parker 1988; Pascoe et al. 2011), and the structuring in the solar corona across the solar disk (Murawski et al. 2001; Yuan et al. 2015, hereafter referred to as Paper I). Nakariakov et al. (2005) examined the dispersive oscillatory wakes of fast waves in randomly structured plasmas. Murawski et al. (2001) studied the possible deceleration of fast waves due to random structuring. Paper I performed a parametric study on the attenuation of fast wave pulses propagating across a randomly structured corona, and proposed the application of the results for seismologically exploiting the frequently observed large-scale extreme ultraviolet (EUV) waves.

Previous investigations of the global EUV waves across the solar disk primarily focused on the nature and properties of the leading front (see the reviews by, e.g., Gallagher \& Long 2011; Patsourakos \& Vourlidas 2012; Liu \& Ofman 2014; Warmuth 2015). However, secondary waves have been observed at strong magnetic waveguides or anti-waveguides, e.g., active regions (Ofman \& Thompson 2002; Shen et al. 2013), coronal holes (Veronig et al. 2006; Li et al. 2012; Olmedo et al.
2012), prominences (Okamoto et al. 2004; Takahashi et al. 2015), and coronal loops (Shen \& Liu 2012). These studies on secondary waves were primarily intended to provide support for the wave nature of EUV waves. However, given that their spatial distribution and temporal evolution can now be observed in substantial detail, secondary waves may well be suitable for remotely diagnosing the structured solar atmosphere as well (Paper I).

In this study, we present a detailed numerical study on the interaction between fast wave pulses with a randomly structured plasma, paying special attention to secondary waves in the wake of the leading fast wave pulse. We describe our numerical model in Section 2, and then present a case study on the secondary waves and their quasiperiodicity in Section 3. Then we perform a parametric study on how this quasi-periodicity is affected by plasma structuring (Section 4). Finally, Section 5 summarizes the present study.

\section{Numerical model}

We used MPI-AMRVAC, a finite-volume code (Keppens et al. 2012; Porth et al. 2014), to solve the ideal MHD equations:

$$
\begin{aligned}
\frac{\partial \rho}{\partial t}+\nabla \cdot(\rho \mathbf{v}) & =0, \\
\frac{\partial \rho \mathbf{v}}{\partial t}+\nabla \cdot\left[\rho \mathbf{v} \mathbf{v}+\mathbf{I} p_{\text {tot }}-\frac{\mathbf{B B}}{\mu_{0}}\right] & =0 \\
\frac{\partial \epsilon}{\partial t}+\nabla \cdot\left[\mathbf{v}\left(\epsilon+p_{\text {tot }}\right)-\frac{(\mathbf{v} \cdot \mathbf{B}) \mathbf{B}}{\mu_{0}}\right] & =0 \\
\frac{\partial \mathbf{B}}{\partial t}+\nabla \cdot(\mathbf{v B}-\mathbf{B v}) & =0,
\end{aligned}
$$

where $\rho$ is the density, $\mathbf{v}$ the velocity, $\mathbf{B}$ the magnetic field, and $\mathbf{I}$ is the unit tensor. In addition, $p_{\text {tot }}=p+B^{2} / 2 \mu_{0}$ is the total pressure, where $p$ is the gas pressure, and $\mu_{0}$ is the magnetic permeability of free space. The total energy density $\epsilon$ is defined by $\epsilon=\rho v^{2} / 2+p /(\gamma-1)+B^{2} / 2 \mu_{0}$, where $\gamma$ is the adiabatic index.

To facilitate the numerical computations, we adopt a set of three independent constants of normalization, namely, $B_{0}=10 \mathrm{G}, L_{0}=1000 \mathrm{~km}$ and $\rho_{0}=7.978 \cdot 10^{-13} \mathrm{~kg} \mathrm{~m}^{-3}$. Some derivative constants are also relevant, e.g., the Alfvén speed $V_{\mathrm{A}}=B_{0} / \sqrt{\rho_{0} \mu_{0}}=1000 \mathrm{kms}^{-1}$ and the Alfvén 
transit time $\tau_{\mathrm{A}}=1 \mathrm{~s}$. In the following text, all symbols represent normalized variables.

The MPI-AMRVAC code was configured to solve the one-dimensional (1D) version of the ideal MHD equations in Cartesian coordinates $(x, y, z)$, meaning that all dependent variables depend only on $y$. We chose the three-step RungeKutta method for time integration. Furthermore, from the multiple finite-volume approaches implemented by the MPI-AMRVAC code, we adopted the HLL approximate Riemann solver (Harten et al. 1983) with the KOREN flux limiter (see, e.g., Tóth \& Odstrčil 1996; Kuzmin 2006).

The equilibrium state, into which fast wave pulses are to be introduced, is characterized by a uniform, $x$-directed magnetic field $\mathbf{B}(t=0, y)=$ $(1,0,0)$. The plasma pressure $p(t=0, y)$ is also uniform, corresponding to a plasma beta of 0.01 everywhere in the computational domain. Random structuring is realized by specifying a proper density profile $\rho(t=0, y)$. A uniform plasma pressure is maintained by adjusting the temperature profile $(p=\rho T)$ accordingly.

The density profile is formed by a set of sinusoidal modulations superimposed on a uniform background,

$$
\rho(t=0, y)=1+\frac{\Delta}{N} \sum_{i=1}^{N} R_{i} \sin \left(\frac{1}{4} \frac{i \pi y}{L_{y}}+\phi_{i}\right),
$$

where $\Delta$ is a scaling parameter, and $L_{y}$ is the size of the numerical domain in the $y$-direction. The values $R_{i}$ and $\phi_{i}$ are the random amplitude and phase of the $i$-th harmonic component, given by uniform pseudo-random number generators within the ranges of $[0,1]$ and $[-\pi, \pi]$, respectively. A correlation length $L_{c}$ is defined to quantify the average spacing between two fine structures; and a density contrast $\delta_{\rho}$ is calculated to define the coarseness of density fluctuations (Paper I). A density distribution with $\delta_{\rho}=0.18$ and $L_{c}=1.26$ is shown in Figure 1(a) for illustration purposes.

Fast wave pulses to be launched into the equilibrium are in the form of a Gaussian profile centered around $y_{0}=0$ with an amplitude of $A_{0}$ and an initial width of $w_{0}$ (Paper I):

$$
\begin{aligned}
& v_{y}^{\prime}(t=0, y)=A_{0} \exp \left[-4 \ln 2 \frac{\left(y-y_{0}\right)^{2}}{w_{0}^{2}}\right], \\
& B_{x}^{\prime}(t=0, y)=A_{0} \exp \left[-4 \ln 2 \frac{\left(y-y_{0}\right)^{2}}{w_{0}^{2}}\right], \\
& \rho^{\prime}(t=0, y)=A_{0} \exp \left[-4 \ln 2 \frac{\left(y-y_{0}\right)^{2}}{w_{0}^{2}}\right],
\end{aligned}
$$

where $v_{y}^{\prime}, B_{x}^{\prime}$, and $\rho^{\prime}$ are the perturbations to the $y$-component of the velocity, the $x$-component of the magnetic field, and plasma density, respectively. This form of perturbation simulates a local eigenmode solution and ensures that fast waves are uni-directional.

Hereafter, fast wave pulses with $A_{0}=0.005$ (0.1) will be referred to as small-(large-) amplitude pulses. As will be shown, the small-amplitude pulses have negligible nonlinear effects, whereas the large-amplitude ones lead to non-restorable density perturbations.

\section{Quasi-periodicity of secondary waves}

We start with an examination of what happens when a small-amplitude fast pulse with $w_{0}=1.78$ is launched into a randomly structured plasma as depicted in Figure 1(a). Figure 1(b) presents the distribution in the $y-t$ plane of the $y$-component of the velocity perturbation $\left(v_{y}\right)$, where the diagonal ridge is the course of the leading fast wave pulse. While propagating, this pulse undergoes a series of interactions with the random plasma, thereby experiencing some attenuation and broadening (Paper I). In addition, secondary waves with amplitudes at about $10-20 \%$ of that of the main pulse are clearly seen, forming a 'fabric' pattern as a result of partial transmissions and reflections. Partial reflection is strong at sharp density changes (e.g., at $y \simeq 20,48,85,105$ ), as evidenced by the strong backward propagation of secondary waves.

We see that the secondary waves exhibit both spatial and temporal quasi-periodicities. To better show this, we derive the the Fourier spectra for the secondary waves shown in the two curves in Figure 1(b) by excluding the main pulse and the zeros ahead of the pulse. As shown in Figure $2(\mathrm{a})$, the velocity variation $v_{y}(t, y=32)$ has prominent oscillations with periods in the range 


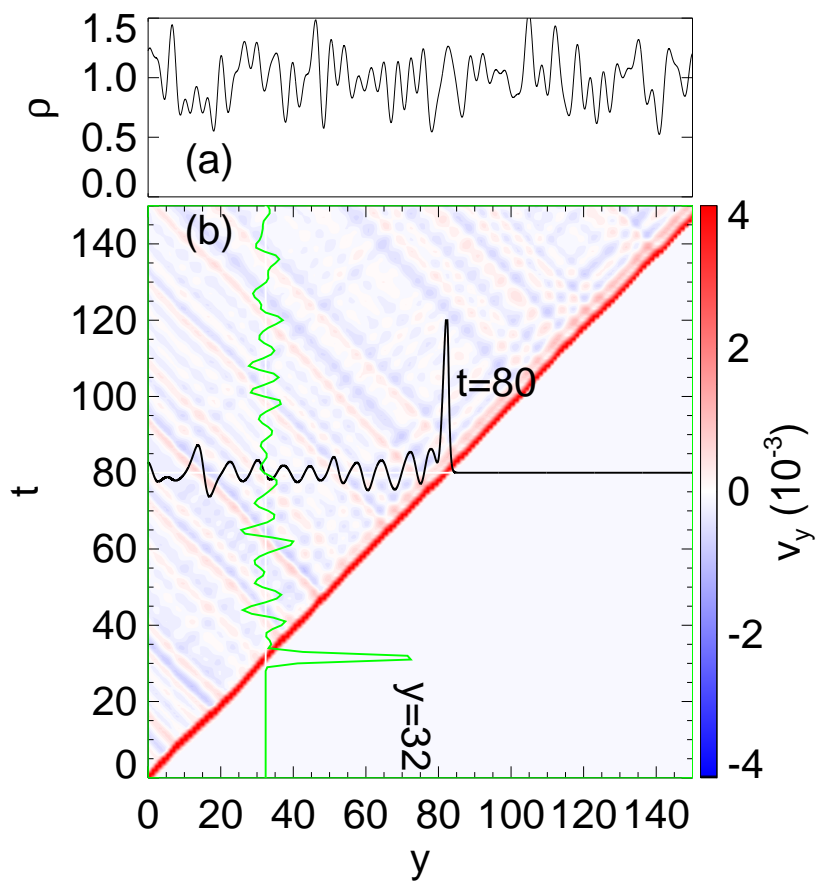

Fig. 1.- (a) Density distribution of a randomly structured plasma with a density contrast $\delta_{\rho}=0.18$ and a correlation length $L_{c}=1.26$. (b) Distribution in the $y-t$ plane of the $y$-component of the velocity perturbation $\left(v_{y}\right)$ in response to a small-amplitude fast pulse with $w_{0}=1.78$. The green (black) curve shows the temporal (spatial) distribution of $v_{y}$ at $y=32(t=80)$. In both curves, the values of $v_{y}$ are multiplied by $10^{4}$ for presentation purposes.

between 6 and 24 (Figure 2(a)). The average period is found to be $\bar{p}=8.5 \pm 2.5$ (see Appendix A for how we calculate this $\bar{p}$ ). This periodicity is not unique to the temporal profile of $v_{y}$ at $y=32$, but is found at all other positions. On the other hand, the spatial period (Figure 2(b)) for $v_{y}(t=80, y)$ ranges from 6 to 22 (or from $5 L_{c}$ to $\left.17 L_{c}\right)$, with an average value of $\bar{\lambda}=8.1 \pm 2.3$. The average spatial period is a few times longer than the correlation length $L_{c}$, meaning that secondary waves need to traverse several correlation lengths before settling into a quasi-periodic signal. This is consistent with Yuan et al. (2016). In addition, the temporal periodicity is found to be correlated with the spatial one, which is not surprising given that the fast wave pulse and secondary waves have an average speed of propagation of about unity in numerical units. The randomness in spatial structuring is transformed into the randomness in the temporal domain. As demonstrated in Yuan et al. (2016), quasi-periodicity is an intrinsic property of a random time series, and the quasi-periods are usually a few times longer than the timescales of the transients.

\section{Parametric Study}

In this section, we investigate how the quasiperiodicities depend on various parameters characterizing the equilibrium density profiles and fast pulses. As is evident from Eq. (5), the equilibrium density profile is characterized primarily by the density contrast $\delta_{\rho}$ and correlation length $L_{c}$. On the other hand, in addition to the amplitudes, fast pulses are also characterized by their initial widths $w_{0}$. To bring out the effects of each individual parameter on the average period $\bar{p}$, we choose to vary a designated parameter alone by fixing the rest. In addition, since the periodicities in the temporal and spatial domains are correlated, we will only show the results for the temporal periodicities. Given that the time series at each position should be sufficiently long to allow the computation of a significant Fourier spectrum, we choose only the $v_{y}$ profiles for locations between $y=10$ to $y=100$. The values for $\bar{p}$ are then scatterplotted for each parameter, see Figures 3, 4, and 5. A smaller spread of period means that secondary waves are trapped by a randomly structured plasma in a more uniform manner, similar to the thermalization of collisional particles heated impulsively.

The dependence of $\bar{p}$ on the density contrast $\delta_{\rho}$ is shown in Figure 3, for which the values of the correlation length and initial pulse width are fixed at $L_{c}=1.26$ and $w_{0}=1.18$, respectively. For small-amplitude pulses, given in Figure 3(a), the mean period does not vary significantly with the density contrast. However, the spread in $\bar{p}$ is larger for larger values of $\delta_{\rho}$. In the case of largeamplitude pulses, shown in Figure 3(b), the spread in $\bar{p}$ tends to be stronger than for small-amplitude pulses. Furthermore, this spread tends to first increase with increasing $\delta_{\rho}$ before decreasing when $\delta_{\rho} \gtrsim 0.2$. This trend can be understood as follows. Large-amplitude pulses can lead to non-restorable 

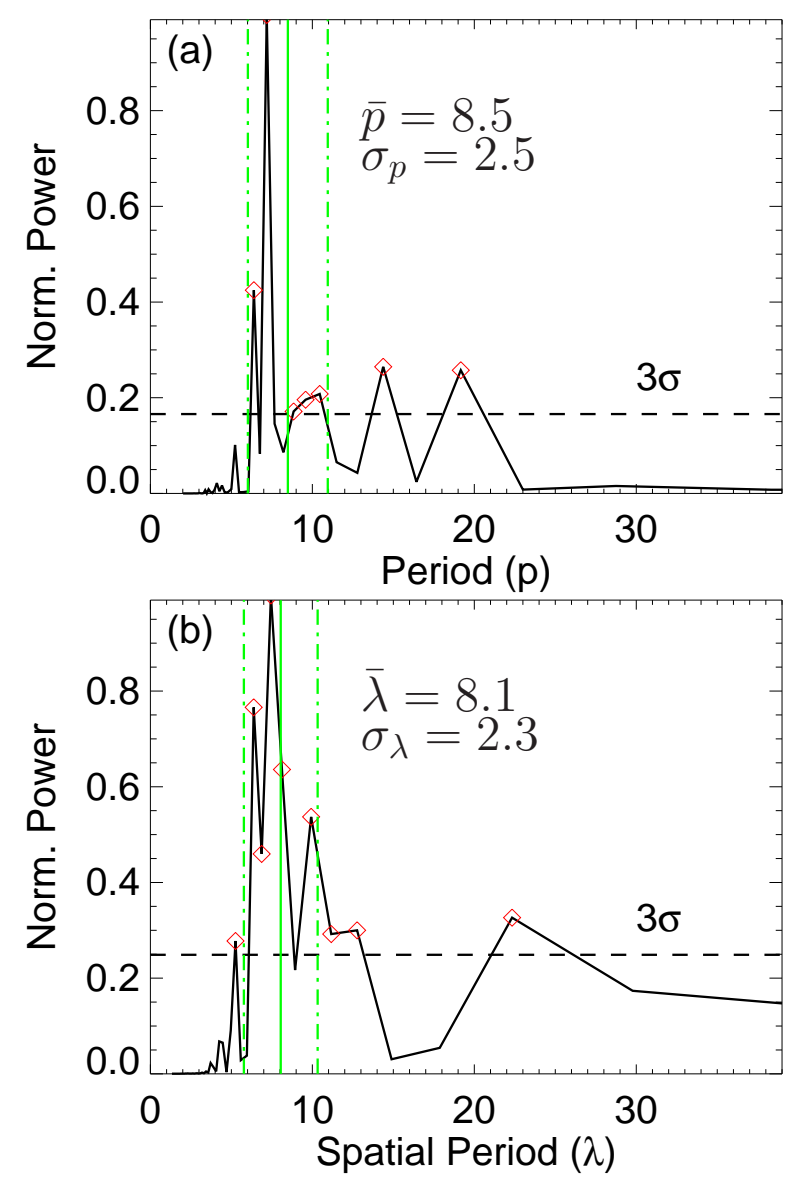

Fig. 2.- Normalized Fourier spectra for (a) $v_{y}(t, y=32)$ and $(\mathrm{b}) v_{y}(t=80, y)$. The solid vertical lines represent the mean periods $(\bar{p}$ and $\bar{\lambda})$, enclosed by the lower and upper limits as given by the dash-dotted lines. The horizontal dashed lines plot the $3 \sigma$ noise level, and the red diamonds mark the frequency components above it. density perturbations (Paper I), and therefore will enhance the density contrast if $\delta_{\rho}$ is weak. However, when $\delta_{\rho}$ is sufficiently strong, the passage of a nonlinear fast wave pulse has a smoothing effect.

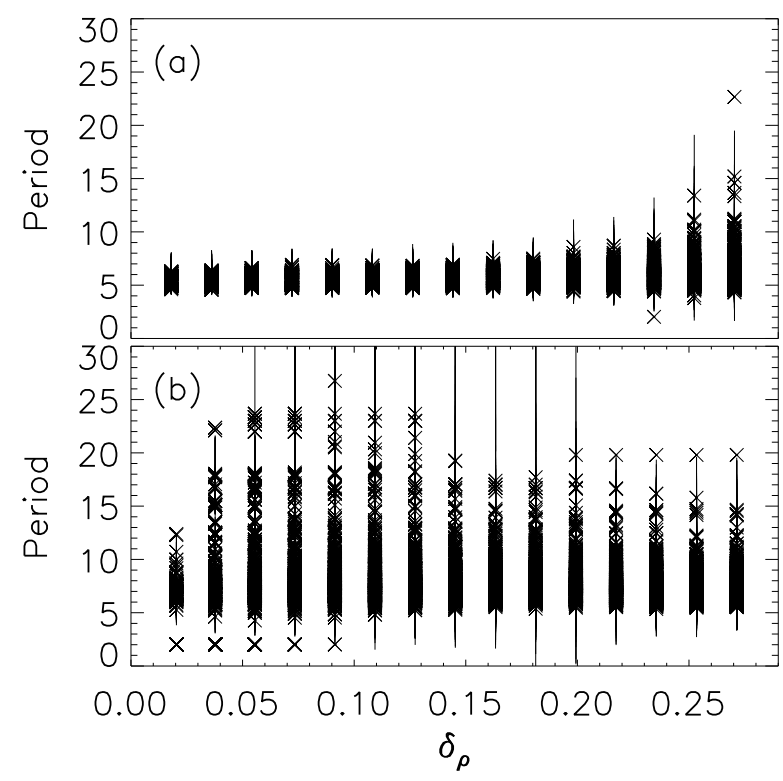

Fig. 3.- Period as a function of density contrast $\delta_{\rho}$ for (a) small and (b) large amplitude pulses. These computations pertain to a fixed correlation length $L_{c}$ of 1.26 , and a fixed initial pulse width $w_{0}$ of 0.18 .

Figure 4 shows the dependence of $\bar{p}$ on the correlation length $L_{c}$. In this set of experiments, we fix the density contrast at $\delta_{\rho}=0.18$ and vary the correlation length. Furthermore, while both pertain to small-amplitude pulses, two different values for the initial pulse width $\left(w_{0}=2.83\right.$ and 1.41) are examined and the results are shown in Figures. 4(a) and 4(b), respectively. It is clear that, regardless of $w_{0}$, the mean period $\bar{p}$ tends to depend linearly on $L_{c}$. Comparing Figures. 4(a) and 4 (b), one sees that a narrow pulse is more sensitive to the variations in the correlation length. The period almost triples as $L_{c}$ doubles. In contrast, the increase in $\bar{p}$ with $L_{c}$ for the broader pulse is not as strong.

How does $\bar{p}$ depend on the initial pulse width $w_{0}$ ? To address this, we launch a set of pulses with different initial widths and investigate their propagation in two randomly structured plasmas with correlation lengths of $L_{c}=2.00$ and 1.26, 


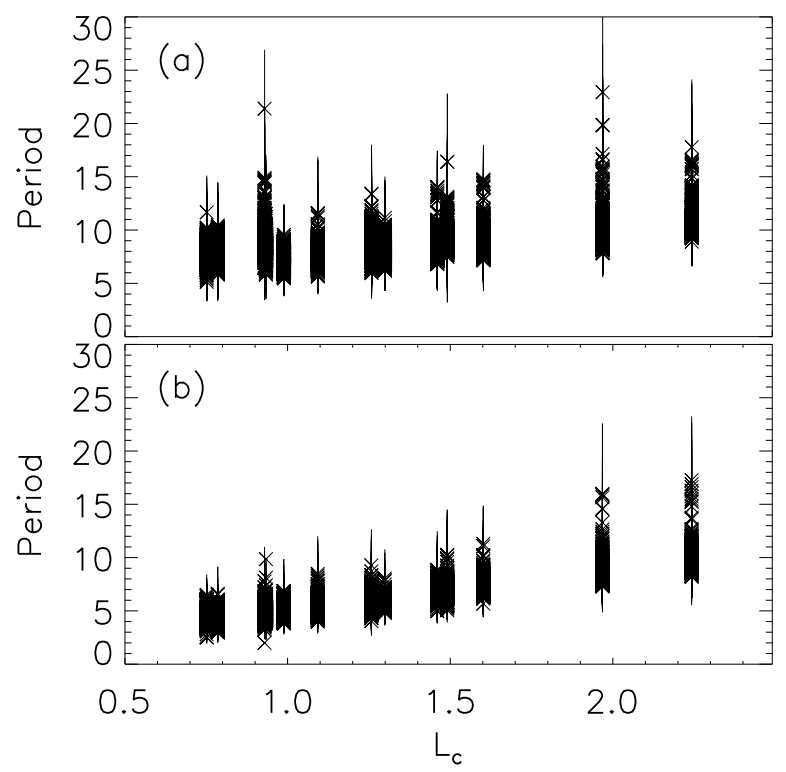

Fig. 4.- Period as a function of correlation length for small-amplitude pulses with two different initial widths: (a) $w_{0}=2.83$ and (b) $w_{0}=1.41$. These computations pertain to a fixed density contrast $\delta_{\rho}$ of 0.18 .

respectively. The resonant energy loss effect (Paper I) is not prominent in the periodicity of the secondary waves. However, Figure 5 shows that broader pulses normally generate secondary waves with longer periods, which is more evident if $L_{c}$ is smaller. This is understandable given that when $L_{c}$ is small, fast pulses will be able to interact with the fine-scale inhomogeneities more frequently.

\section{Conclusions}

This study offered a series of numerical simulations on the propagation of fast MHD wave pulses in randomly structured plasmas mimicking the solar corona. While traversing the plasma inhomogeneities, fast wave pulses experience partial reflections, giving rise to secondary waves propagating in the opposite direction. In turn, these waves generate further waves, once again due to their partial reflection and transmission at plasma inhomogeneities. The end result is that, the energy contained in the primary fast pulses is spread over the randomly structured plasmas in the form of propagating and standing sec-

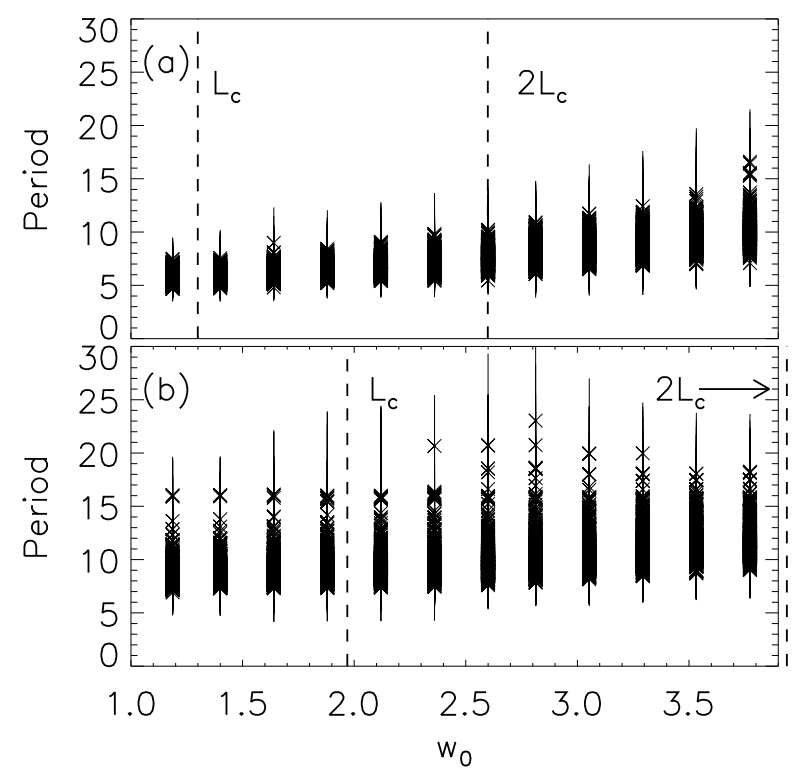

Fig. 5.- Period as a function of initial pulse width. Two density profiles with correlation lengths of (a) $L_{c}=1.26$ and (b) $L_{c}=2.0$ are examined, even though both pertain to the same density contrast of $\delta_{\rho}=0.18$. The vertical dashed lines mark where the initial pulse width matches $L_{c}$ and $2 L_{c}$.

ondary waves. These secondary waves exhibit quasi-periodicities in both time and space. The spatial period at a given instant is related to the period observed at a fixed position via the fast wave speed.

The interaction between fast wave pulses and the plasma inhomogeneities, as quantified by the average temporal period $\bar{p}$ of secondary waves, turns out to depend primarily on the combination of parameters $\left[\delta_{\rho}, L_{c}, w_{0}\right]$. Here $\delta_{\rho}$ and $L_{c}$ are the density contrast and correlation length that characterize the equilibrium density profile. Furthermore, $w_{0}$ is the initial width of the wave pulse. For small-amplitude pulses, $\delta_{\rho}$ does not have a significant effect on $\bar{p}$. Rather, it determines the rapidness for $\bar{p}$ to reach a uniform distribution. Large-amplitude pulses, on the other hand, lead to non-restorable density perturbations, thereby enhancing the density contrast when $\delta_{\rho}$ is small but having a smoothing effect when $\delta_{\rho}$ is sufficiently large. The average period $\bar{p}$ scales linearly with the correlation length, with the scaling factors be- 
ing larger for narrower pulses. However, broader pulses can generate secondary waves with longer periods, the effect being stronger in random plasmas with shorter correlation lengths.

Secondary waves carrying the signatures of both the leading wave pulse and background plasma may be detected in the dimming regions after CMEs or EUV waves (see Guo et al. 2015; Chandra et al. 2016, for some recent observations). However, a dedicated observational study is needed to fully explore the seismological applications of the present study.

We thank the anonymous referee for the constructive comments. This work is supported by the Open Research Program KLSA201504 of Key Laboratory of Solar Activity of National Astronomical Observatories of China (DY). It is also supported by the National Natural Science Foundation of China $(41174154,41274176$, and 41474149).

\section{REFERENCES}

Arregui, I., Andries, J., Van Doorsselaere, T., Goossens, M., \& Poedts, S. 2007, A\&A, 463, 333

Aschwanden, M. J., Nightingale, R. W., Andries, J., Goossens, M., \& Van Doorsselaere, T. 2003, ApJ, 598, 1375

Chandra, R., Chen, P. F., Fulara, A., Srivastava, A. K., \& Uddin, W. 2016, ApJ, 822, 106

Chen, Y., Feng, S. W., Li, B., et al. 2011, ApJ, 728,147

Chen, Y., Song, H. Q., Li, B., et al. 2010, ApJ, 714,644

De Moortel, I., \& Nakariakov, V. M. 2012, Royal Society of London Philosophical Transactions Series A, 370, 3193

Edwin, P. M., \& Roberts, B. 1983, Sol. Phys., 88, 179

Gallagher, P. T., \& Long, D. M. 2011, Space Sci. Rev., 158, 365

Goedbloed, J. P., \& Poedts, S. 2004, Principles of magnetohydrodynamics: with applications to laboratory and astrophysical plasmas (Cambridge university press)

Guo, Y., Ding, M. D., \& Chen, P. F. 2015, ApJS, 219,36

Harten, A., Lax, P. D., \& Leer, B. v. 1983, SIAM review, 25,35

Heyvaerts, J., \& Priest, E. R. 1983, A\&A, 117, 220

Keppens, R., Bogdan, T. J., \& Goossens, M. 1994, ApJ, 436, 372

Keppens, R., Meliani, Z., van Marle, A. J., et al. 2012, Journal of Computational Physics, 231, 718

Kuzmin, D. 2006, Journal of Computational Physics, 219, 513

Li, T., Zhang, J., Yang, S., \& Liu, W. 2012, ApJ, 746,13

Liu, W., \& Ofman, L. 2014, Sol. Phys., 289, 3233

Murawski, K., Nakariakov, V. M., \& Pelinovsky, E. N. 2001, A\&A, 366, 306

Nakariakov, V. M., \& Ofman, L. 2001, A\&A, 372, L53

Nakariakov, V. M., Pascoe, D. J., \& Arber, T. D. 2005, Space Sci. Rev., 121, 115

Nakariakov, V. M., \& Verwichte, E. 2005, Living Reviews in Solar Physics, 2, 3

Ofman, L., \& Thompson, B. J. 2002, ApJ, 574, 440

Okamoto, T. J., Nakai, H., Keiyama, A., et al. 2004, ApJ, 608, 1124

Olmedo, O., Vourlidas, A., Zhang, J., \& Cheng, X. 2012, ApJ, 756, 143

Parker, E. N. 1988, ApJ, 330, 474

Pascoe, D. J., Wright, A. N., \& De Moortel, I. 2011, ApJ, 731, 73

Patsourakos, S., \& Vourlidas, A. 2012, Sol. Phys., 281, 187

Porth, O., Xia, C., Hendrix, T., Moschou, S. P., \& Keppens, R. 2014, ApJS, 214, 4 
Sakurai, T., Goossens, M., \& Hollweg, J. V. 1991, Sol. Phys., 133, 227

Shen, Y., \& Liu, Y. 2012, ApJ, 754, 7

Shen, Y., Liu, Y., Su, J., et al. 2013, ApJ, 773, L33

Takahashi, T., Asai, A., \& Shibata, K. 2015, ApJ, 801,37

Torrence, C., \& Compo, G. P. 1998, Bulletin of the American Meteorological Society, 79, 61

Tóth, G., \& Odstrčil, D. 1996, Journal of Computational Physics, 128, 82

Van Doorsselaere, T., Brady, C. S., Verwichte, E., \& Nakariakov, V. M. 2008, A\&A, 491, L9

Van Doorsselaere, T., Wardle, N., Del Zanna, G., et al. 2011, ApJ, 727, L32

Veronig, A. M., Temmer, M., Vršnak, B., \& Thalmann, J. K. 2006, ApJ, 647, 1466

Warmuth, A. 2015, Living Reviews in Solar Physics, 12, doi:10.1007/lrsp-2015-3

Yuan, D., Nakariakov, V. M., Huang, Z., et al. 2014a, ApJ, 792, 41

Yuan, D., Pascoe, D. J., Nakariakov, V. M., Li, B., \& Keppens, R. 2015, ApJ, 799, 221

Yuan, D., Su, J., Jiao, F., \& Walsh, R. W. 2016, ApJS, 224, 30

Yuan, D., Sych, R., Reznikova, V. E., \& Nakariakov, V. M. 2014b, A\&A, 561, A19

Zhang, Y., Zhang, J., Wang, J., \& Nakariakov, V. M. 2015, A\&A, 581, A78

This 2-column preprint was prepared with the AAS LATEX macros v5.2. 


\section{A. Calculation of quasi-periodicity}

We use $f(s)$ to represent either the spatial distribution of the velocity distribution $v_{y}(y, t)$ at a fixed instant or its temporal variation at a fixed location, after excluding both the leading pulse and the zeros ahead. The Fourier transform $\mathscr{F}(\xi)$ is calculated as

$$
\mathscr{F}(\xi)=\int_{-\infty}^{\infty} f(s) e^{-2 \pi j s \xi} \mathrm{d} s
$$

where $j=\sqrt{-1}$, and $\xi$ denotes either the spatial or temporal frequency. As a result, $1 / \xi$ will be either the spatial or temporal period. The Fourier spectrum is then obtained by calculating $P(\xi)=|\mathscr{F}(\xi)|^{2}$.

The mean frequency $\bar{\xi}$ is taken to be a weighted average,

$$
\bar{\xi}=\frac{\int_{-\infty}^{\infty} P(\xi) \xi \mathrm{d} \xi}{\int_{-\infty}^{\infty} P(\xi) \mathrm{d} \xi} .
$$

The error bar $\sigma_{\xi}$ is then computed by using

$$
\sigma_{\xi}^{2}=\frac{\int_{-\infty}^{\infty} P(\xi) \xi^{2} \mathrm{~d} \xi}{\int_{-\infty}^{\infty} P(\xi) \mathrm{d} \xi}-\bar{\xi}^{2}
$$

In our calculations, we integrate only the frequency components with power above the $3 \sigma$-noise level (see Torrence \& Compo 1998, for its definition). In terms of the temporal or spatial periods, we adopt $1 / \bar{\xi}$ and $\sigma_{\xi} / \bar{\xi}^{2}$ as the mean value and its associated uncertainty (see the vertical lines in Figure 2). 